\title{
Supportive tissues of the vagina with special reference to a fibrous skeleton in the perineum: A review
}

\author{
Hiromasa Sasaki $^{1}$, Nobuyuki Hinata ${ }^{2 *}$, Tetsuji Kurokawa ${ }^{3}$, Gen Murakami ${ }^{4,5}$ \\ ${ }^{1}$ Division of Gynecology and Obsterics, Ishikawa Prefectural Central Hospital, Kanazawa, Japan \\ ${ }^{2}$ Department of Urology, Kobe University Graduate School of Medicine, Kobe, Japan \\ ${ }^{3}$ Department of Obstetrics and Gynecology, Fukui University School of Medicine, Fukui, Japan \\ ${ }^{4}$ Division of Internal Medicine, Iwamizawa Kojin-kai Hospital, Iwamizawa, Japan \\ ${ }^{5}$ Department of Anatomy, Tokyo Dental College, Chiba, Japan \\ Email: "hinata@med.kobe-u.ac.jp
}

Received 15 December 2013; revised 12 January 2014; accepted 20 January 2014

Copyright (C) 2014 Hiromasa Sasaki et al. This is an open access article distributed under the Creative Commons Attribution License, which permits unrestricted use, distribution, and reproduction in any medium, provided the original work is properly cited. In accordance of the Creative Commons Attribution License all Copyrights @ 2014 are reserved for SCIRP and the owner of the intellectual property Hiromasa Sasaki et al. All Copyright @ 2014 are guarded by law and by SCIRP as a guardian.

\section{ABSTRACT}

With the aid of immunohistochemistry, the present review attempts to demonstrate the composite fibers and nerve topographical anatomy in the vaginal supportive tissues. Along the tendinous arch of the pelvic fasciae, distal parts of the pelvic plexus extend antero-inferiorly and issue nerves to the internal anal sphincter as well as the cavernous tissues. At the attachment of the levator ani muscle to the rectum, smooth muscles in the endopelvic fascia lining the levator ani merge with the longitudinal smooth muscle layer of the rectum to provide the conjoint longitudinal muscle coat or the longitudinal anal muscle (LAM: smooth muscle). However, at the rectovaginal interface, the longitudinal smooth muscle layer of the rectum continues to the LAM without any contribution of the endopelvic fascia. The bilateral masses of the perineal smooth muscles (PSMs) are connected by the perineal body, and the PSMs receive 1) the longitudinal anal muscle, 2) the internal and external anal sphincters and, 3) the perineal membrane lining the vestibular wall. Tensile stress from the levator ani seems to be transferred to the PSMs via the LAM. Because of their irregularly arrayed muscle fibers, instead of a synchronized contraction in response to nerve impulses, the PSMs are likely to act as a barrier, septum or protector against mechanical stress because, even without innervation, such smooth muscle fibers resist (not absorb) pressure, in accordance with Bayliss' rule. The external anal sphincter, a strong striated muscle, inserts into the PSMs and seems to

\footnotetext{
*Corresponding author.
}

play a dynamic role in supporting the rectovaginal interface to maintain the antero-posterior length of the urogenital hiatus. However, we do not think that smooth muscles play an active traction role without cooperation from striated muscle. The fibrous skeleton composed of smooth muscle in the female perineum is explained in terms of a “catamaran” model.

\section{KEYWORDS}

Vagina; Levator Ani Muscle; Perineal Membrane; Smooth Muscles; Elastic Fibers; Nerves; Pelvic Floor Anatomy

\section{INTRODUCTION}

The vaginal support structures comprise a series of connective tissues that are well known to gynecologists, i.e., levels I, II and II described by DeLancey [1-3]. However, few studies have attempted to demonstrate the configuration of composite fibers in these tissues. In order to consider the transfer of tensile stress from the vagina to the pelvic floor and wall, we believe that a detailed knowledge of the morphology of the composite fibers is necessary. In particular, since the initial report by Oelrich [4], there have been very few histological demonstrations of level III support. Soga et al. [5] demonstrated a distinct connective tissue mass in the lateral side of the distal vagina, which they termed a difficult name, the lateral extension of the perineal body. They considered that the connective tissue, comprising mainly smooth muscles alongside the vestibule, is located adjacent to and connects with both the external and internal anal sphincters, thus suggesting functional cooperation between the va- 
ginal support and the anal sphincter.

Oelrich [4] considered the female perineal smooth muscles to be a membranous structure corresponding to the male urogenital diaphragm. In a histological section covering a small area, Hudson et al. [6] identified several fiber bundles as the perineal membrane; however, this was beyond the scope of a "topohistological" demonstration. Using histology and macroscopic slices, Stein and DeLancey [7] found that both the compressor urethrae and urethrovaginal sphincter are closely "associated" with the female perineal membrane. However, in their photos, it was difficult to identify the composite fibers of the membrane. Unlike our interpretation, they considered the perineal membrane to connect with the endopelvic fascia (fascia pelvis parietalis) covering the internal aspect of the levator ani muscle. In the same year, Kato et al. [8] clearly demonstrated that elastic fibers between the urethral rhabdosphincter muscle fibers join together to form the female perineal membrane. Thus, an elastic fiber cage for the urethral rhabdosphincter, which is evident in males [9], seems to be a common feature in both genders. However, the topographical relationship between the female perineal membrane and the perineal smooth muscles has not been demonstrated. Consequently, with an aid of immunohistochemistry, the present review attempts to demonstrate the topohistology of the supportive tissues of the vagina, especially the most distal part. In addition, we focused on nerves passing in and along the vaginal supportive tissues, as nerves in the lower paracolpium seem to correspond to the distal part of the pelvic autonomic nerve plexus [10-13].

\section{MATERIALS AND METHODS}

This study was performed in accordance with the provisions of the Declaration of Helsinki 1995 (as revised in Edinburgh 2000). The histological sections shown in this review were obtained from 10 donated female cadavers ranging in age from 78 to 96 years, with a mean age of 88 years. The cause of death had been ischemic heart failure or intracranial bleeding, and we confirmed that none of the individuals had undergone surgery by reference to medical documentation as well as macroscopic observation after opening the abdominopelvic cavity. These cadavers had been donated to Tokyo Dental College for research and education on human anatomy, and their use for research had been approved by the university ethics committee. The cadavers had been fixed by arterial perfusion with $10 \% \mathrm{v} / \mathrm{v}$ formalin solution and stored in $50 \% \mathrm{v} / \mathrm{v}$ ethanol solution for more than 3 months.

After routine procedures for paraffin-embedded histology, most sections were subjected to hematoxylin and eosin (HE), azan, Masson trichrome or silver staining, and some were used for immunohistochemistry as well as elastica-Masson staining (a variation of Masson-Goldner staining) or aldehyde fuchsin staining for elastic fibers. The primary antibodies used for nerve immunohistochemistry were 1) mouse monoclonal anti-human S100 protein (1:200 dilution; Dako Z0311; Dako, Glostrup, Denmark), 2) rabbit polyclonal anti-human neuronal nitric oxide synthase (nNOS) (1:200; Cell Signaling Technology, Beverly, MA), 3) mouse monoclonal anti-human vasoactive intestinal polypeptide (H-6 or VIP) (1:100 dilution; Santa Cruz sc25347; Santa Cruz, CA) and 4) rabbit polyclonal anti-human tyrosine hydroxylase (TH) (1:100; Millipore-Chemicon ab152, Temecula, CA). In addition, for fibrous structures, we used mouse monoclonal anti-human alpha smooth muscle actin (1:100; Dako M0851, Glostrup, Denmark) and mouse monoclonal anti-human desmin (dilution, 1:50; Dako N1526). The secondary antibody was labeled with horseradish peroxidase (HRP), and antigen-antibody reactions were detected by the HRP-catalyzed reaction with diaminobenzidine. Counterstaining with hematoxylin was performed on the same samples. A negative control without a primary antibody was set up for each of the specimens. Observations and photography were usually performed with a Nikon Eclipse 80, but photos at ultra-low magnification (objective lens less than $\times 2$ ) were taken using a high-grade flat scanner with translucent illumination (Epson scanner GTX970).

\section{LEVATOR ANI MUSCLE, COLLAGENOUS FIBERS AND ELASTIC FIBERS}

In the pelvic floor, the strongest striated (or skeletal) muscle contraction is generated by the levator ani muscle. It is perhaps pertinent to begin this review by considering the specificity of the levator ani from the viewpoint of the composite fibers in and around the striated muscle (Figure 1). In the human body, striated muscle cells or fibers are surrounded by the endomysium, which comprises type IV and other collagens, whereas intramuscular and extramuscular tendons are composed of type I collagen [14-17] (Figure 1(A) insert). Typical striated muscle fibers carry a series of specific collagenous structures (a form of enthesis [18]) to conduct the force of contraction to a bone or ligament via a tendon. In contrast, the levator ani insertion is formed by an elastic fiber-smooth muscle complex (Figures 1(C) and (D)) classically termed the "conjoint longitudinal muscle coat" [19].

The term "conjoint" was given to the muscle insertion because it is formed by joining between 1) the longitudinal smooth muscle layer of the rectum and 2) the other smooth muscles in the endopelvic fascia covering the internal aspect of the levator: Arakawa et al. [20] and 


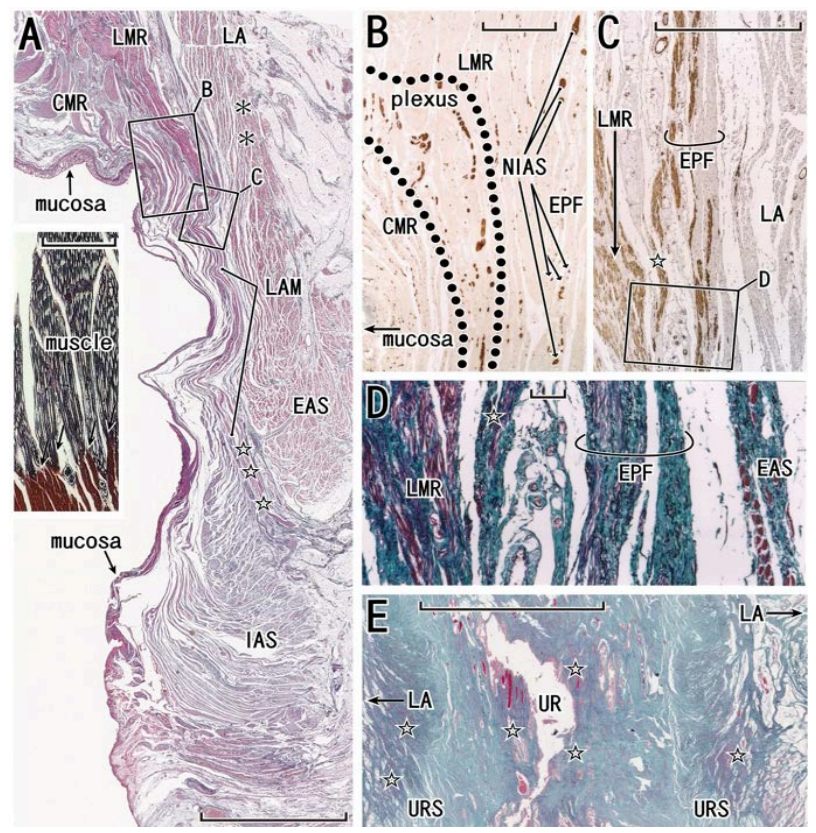

Figure 1. Introduction of the muscle-tendon interface: a skeletal muscle, the longitudinal anal muscle and the female urethra. Panels A-D, frontal sections of specimens from a 78-year-old woman (the anterior part of the specimen will be shown in Figure 7). Panel E, frontal section of a 75-year-old woman. Panels A, D and E show elastica-Masson staining (striated muscles, red; collagen fibers, green; elastic fibers, black or blue). Panel B (or C) exhibits immunohistochemistry for all nerves (or of smooth muscle fibers) at the attaching site of the levator ani (LA) to the rectum. In panel A, the longitudinal muscle layer of the rectum (LMR) continues to the longitudinal anal muscles (LAM) passing through the intersphincteric space (stars) between the external and internal anal sphincters (EAS, IAS). The levator ani is connected with the external anal sphincter by a fascial structure interposed (asterisks). Insert in panel A (silver staining of a skeletal muscle) displays the typical interface (arrows) between the striated muscle fiber (black color, mainly type IV collagen) and the intramuscular tendon (red-brown color, mainly type I collagen). Panel B, corresponding to a square with $B$ in panel $A$, contains the intrinsic nerves of the rectum (surrounded by dotted line; plexus) as well as the extrinsic nerves to the rectum (NIAS) coming along the endopelvic fascia (EPF). Panel $C$, corresponding to a square with $C$ in panel A, exhibits composite smooth muscle fibers in the endopelvic fascia. Panel D, corresponding to a square with $\mathrm{D}$ in panel C, show elastic fibers (black wavy lines) associated with smooth muscles. In panels C and D, a fiber bundle (star), comprising of smooth muscles, elastic fibers and collagen fibers, is originates from the endopelvic fascia and merges with the longitudinal muscle layer of the rectum. Panel E displays high content of elastic fibers (stars) in and along the urethral wall. Scale bars: $10 \mathrm{~mm}$ in panels A and E; $1 \mathrm{~mm}$ in panels B and C; $0.1 \mathrm{~mm}$ in panel D and the insert in panel A. CMR, circular muscle layer of the rectum; NIAS, nerves to the internal anal sphincter; plexus, nerves in the myenteric plexus of the rectum; URS, urethral rhabdosphincer area of the urethra; UR, urethra.

Hieda et al. [21] clearly demonstrated the conjoint or combined connective tissue fibers. However, as the his- tology has not been well clarified, its context in pelvic anatomy has often been ignored or misunderstood (for details, see the final paragraph in the subsection entitled "Perineal smooth muscles"). In this context, a new term that does not involve the concept of joining fibers has recently been adopted, i.e., the "longitudinal anal muscle" or LAM [22]. We have chosen the latter term in this review for the convenience of readers. The LAM seems to provide a "fibrous skeleton" for the anal sphincters to minimize any damage or tears resulting from sudden and strong contraction of the levator ani. The major part extends through the intersphincteric space toward the anal skin and minor parts penetrate the subcutaneous part of external sphincters. Figure 1(A) displays a poorly developed LAM without the minor parts dividing the external anal sphincter as intramuscular septa.

Collagen fibers (type I collagen fibers) possess very little elasticity, whereas elastic fibers absorb tensile stress and recover their length. Tendons of skeletal muscles, which are composed of mostly type I collagen fibers, require a small proportion of elastic fibers in order to recover their length after muscle contraction [23,24]. However, as typically seen in extremities, the covering fascia of a striated muscle contains few or no elastic fibers, because its elastic nature is due largely to the mesh structure of its constituent collagen fibers [25] and partly to elasticity of the muscle cells thmselves [26]. Fasciae that are exceptionally elastic fiber-rich cover or bundle striated muscle fibers in the extra-ocular muscles of the eye [27], the intrinsic lingual muscles along the tongue surface [28], and also the levator ani muscle. Fasciae of the levator ani, including the endopelvic fascia, are rich in both elastic fibers and smooth muscles (Figures 1(C), (D), 2 and 3), especially at the inferomedial edge facing the urethral rhabdosphincter [29].

Other than striated muscles, elastic fiber-mediated connections have been described in the larynx [30,31], the middle ear [32] and the urethra [9] (Figure 1(E)).

According to Hinata et al. [9], the urethra in males contains a much greater amount of elastic fibers than the female counterpart. At these three sites, in combinationwith hyaluronic acid as a lubricant, elastic fibers seem to avoid damage at their connection point when subjected to vibration or drastic changes in shape. Notably, the female endopelvic fascia is characterized by a rich content of elastic fibers [33], possibly resulting from the difference in hormonal backgrounds, as estrogen is known to increase the formation of elastic fibers [34].

The most striking difference between the levator ani and other striated muscles lies in the relationship between the direction of muscle action and that of the muscle fibers: in skeletal muscle, the muscle fibers, tendon and fibrous tissue connecting these are consistently arranged "in series" along an almost straight line (Figure 4(A)), 


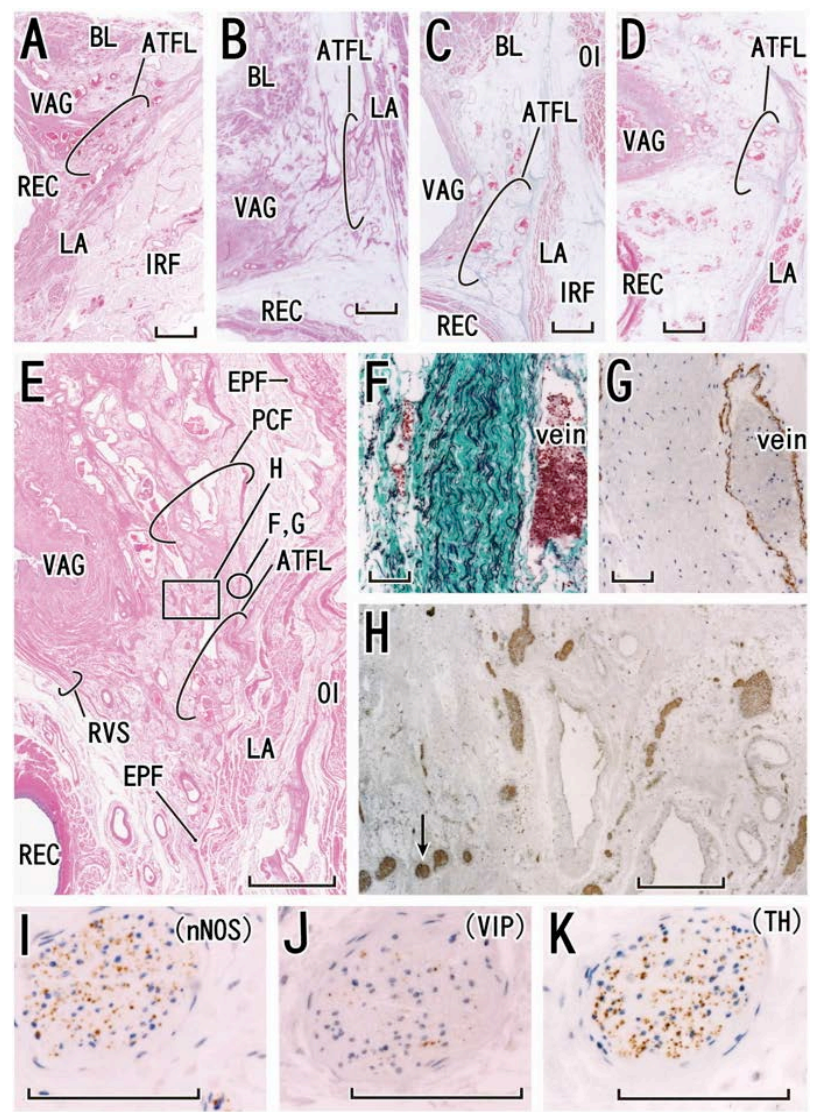

Figure 2. Histology of the tendineous arch of the pelvic fasciae. Tilted frontal sections. Panel A (HE staining, an 85-year-old woman; thick and bundled fasciae), panel B (HE staining, an 82-year-old woman; thick and solid fasciae), panel C (Masson-trichrome staiuning, an 87-year-old woman: few thick fasciae), panel D (Masson-trichrome staining, an 81-year-old woman; unclear fasciae) and, panel E (HE staining, a 79-year-old woman; thick and loose fasciae) display variations in the fascial configuration at the tendineous arch (scale bar in panels A-E, $10 \mathrm{~mm}$ ). Panels E-K show near sections. Panel F (elastica-Masson staining; elastic fibers, black) and panel G (immunohistochemistry for smooth muscles) exhibit a part of the pubocervical fascia (PCF) corresponding to a circle in panel E (scale bars in panels $F$ and $G, 0.1 \mathrm{~mm}$ ). The fascia contained abundant elastic fibers but no smooth muscles. Panel H (immunohistochemistry for all nerves; scale bar, $1 \mathrm{~mm}$ ), corresponding to a square in panel $\mathrm{E}$, displays nerves embedded in the tendineous arch. One of the nerves (arrow in panel $\mathrm{H}$ ) is shown in panel I (immunohistochemistry for parasympathetic nerve fibers; neuronal nitric oxide synthase or nNOS), panel J (immunohistochemistry for parasympathetic nerve fibers; vasoactive intestinal polypeptide or VIP) and panel K (immunohistochemistry for sympathetic nerve fibers). Scale bars in panels I, $\mathrm{J}$ and $\mathrm{K}$ are $0.1 \mathrm{~mm}$. ATFL, arcus tendineous fasciae pelvis; BL, bladder; EPF, endopelvic fascia; IRF, ischiorectal fossa; LA, levator ani muscle; OI, obturator internus muscle; REC, rectum; RVS, rectovaginal septum or Denonvilliers' fascia; VAG, vagina.

whereas the levator muscle fibers are not directed to the urethra and vagina (Figures 4(C) and (D)). A mesh-like

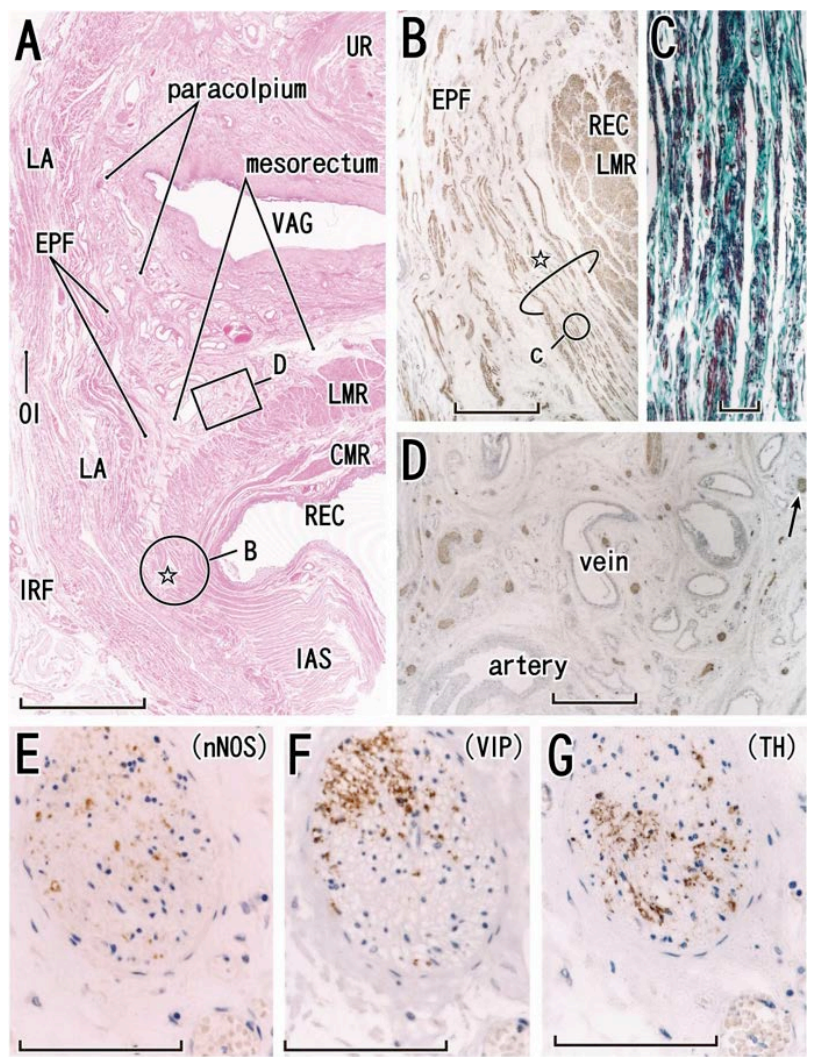

Figure 3. Histology of the lower paracolpium in a specimen from a 73-year-old woman. Tilted frontal sections. Panel A displays topographical anatomy of the paracolpium with HE staining (scale bar, $10 \mathrm{~mm}$ ). Panel B (immunohistochemistry for smooth muscles; scale bar, $1 \mathrm{~mm}$ ), corresponding to a circle in panel A, displays a smooth muscle bundle (star) from the endopelvic fascia (EPF) to join the longitudinal muscle layer of the rectum (LMR). Panel C (elastica-Masson staining; elastic fibers, black), corresponding to a circle in panel B, exhibits abundant elastic fibers in the conjoint smooth muscle bundle (scale bar, $0.1 \mathrm{~mm}$ ). Panel D (immunohistochemistry for all nerves), corresponding to a square in panel A, shows abundant nerves in the paracolpium or mesorectum (scale bar, $1 \mathrm{~mm}$ ). The paracolpium is continuous with the mesorectum without a clear demarcation. One of the nerves (arrow in panel D) is shown in panel E (immunohistochemistry for parasympathetic nerve fibers; neuronal nitric oxide synthase or nNOS), panel F (immunohistochemistry for parasympathetic nerve fibers; vasoactive intestinal polypeptide or VIP) and panel G (immunohistochemistry for sympathetic nerve fibers). Scale bars in panels E-G are $0.1 \mathrm{~mm}$. BL, bladder; CMR and LMR, circular and longitudinal muscle layers of the rectum (smooth muscles); $\mathrm{EPF}$, endopelvic fascia; IAS, internal anal sphincter; IRF, ischiorectal fossa; LA, levator ani muscle; OI, obturator internus muscle; REC, rectum; VAG, vagina.

fascial structure lying between the levator ani and vagina (Figures 2(E) and 3(A)) would also seem to play a role in 1) stabilizing structures in the event of elevation force, and 2) regulating and distributing tensile stress from the levator ani. However, rather than a mechanical role, the levator-vagina interface acts as a major nerve pathway to 


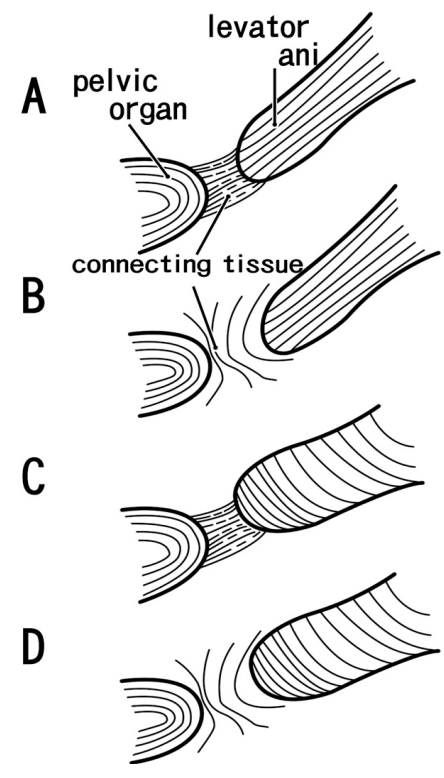

Figure 4. Diagram of interface tissue configurations between the pelvic organ and levator ani muscle. In panels A and B, muscle fibers of the levator ani directing inferomedially are suitable for upward traction of the organ, whereas in panels $\mathrm{C}$ and $\mathrm{D}$ the fiber directions of the oblique muscle are not suitable for such traction. In panels A and C, the fibrous tissues or fasciae connecting between the levator ani and organ are arranged in series along the axis from the levator to the organ, whereas in panels $\mathrm{B}$ and $\mathrm{D}$, the fasciae along or surrounding the levator and/or the organ are not suitable for force transduction but for sliding between these two. The morphology shown in panel A, being similar to the muscle-tendon interface in a skeletal muscle (see Figure 1(A) insert), is most suitable for upward traction. The levator ani (strictly, the endopelvic fascia) and the rectum exhibit this type of morphology, but the connecting tissues contain abundant elastic fibers and smooth muscle (see Figures 1(C) and (D)). The morphology shown in panel B is similar to an interface between the levator ani and external anal sphincter (see Figure 1(A)). Panel C displays the interface between the levator ani and urethral rhabdosphincter (see Figure $1(\mathrm{E})$ ). Panel D corresponds to the configuration between the levator and vagina.

the rectum (see the subsection below). Therefore, any direct transfer of force from the levator to the vagina would seem to be limited.

\section{TENDINOUS ARCH OF THE PELVIC FASCIA AND THE PARACOLPIUM}

The tendinous arch of the pelvic fascia, or the so-called "white line", has been well studied in terms of gross anatomy [35-38]. However, the histology does not exhibit a "linear" structure but a mesh-like complex of multiple fasciae [12,33,39] (Figures 2(A)-(E)). Along the tendinous arch, the rectovaginal septum and pubocervical fascia meet the endopelvic fascia [2]. Both are composed of elastic and collagenous fibers and they contain no or few smooth muscles [40] (Figures 2(F) and (G)). Al- though it is not commonly known, the tendinous arch and its associated loose connective tissue (i.e., the paracolpium) is one of the striking anatomical features in the female pelvic floor. Because of the bulky mass of the prostate, the periprostatic tissue along the endopelvic fascia is very narrow, without any definite tendinous arch from a histologic viewpoint $[33,41]$. The morphology of the tendinous arch varied between individuals (Figures 2(A)-(E)), possibly due to age-related degeneration and damage sustained during vaginal delivery [42]. Recently, celloidin-embedded large sections have been used for demonstration of the pelvic fasciae [43], but these may not be applicable for immunohistochemistry because of their thickness. Abundant nerves run antero-inferiorly along the tendinous arch and pass through small spaces divided by multiple fasciae continuous with either or both the rectovaginal septum and pubocervical fascia (Figure 2(H)). Thus, the nerves cross the composite fibers of the fasciae.

Nerves in the lower paracolpium provide the origins of nerves extending to the internal anal sphincter $[13,21]$ as well as the cavernous nerves $[42,44]$. In view of the origins of these nerves, the posterior half of the lower paracolpium seems to correspond to a nerve-rich space at the posterolateral corner of the prostate in males. The rectovaginal septum corresponds to Denonvilliers' fascia in females [43]. Thus, as in males [45,46], the rectovaginal septum provides the anterior boundary of a fatty tissue layer surrounding the rectum, i.e., the mesorectum [47]. However, in elderly individuals, because the septum is usually fragmented or unclear in the lateral area, the lower paracolpium becomes continuous with the mesorectum without any clear demarcation (Figure 3(A)).

Using three markers (nNOS and VIP for parasympathetic nerves; TH for sympathetic nerves; for abbreviations see Materials and Methods), Hieda et al. [48] found four combinations of autonomic motor nerve fibers in the lower paracolpium: [nNOS+, VIP+, TH+], $[+,-,+],[-$, $-,+]$ and $[-,-,-]$, not 8 , which would logically be the largest number of combinations (i.e., $2 \times 2 \times 2$ ). Nerves negative for all three markers, i.e., $[-,-,-]$, were composed of thick, myelinated sensory fibers. The first and second patterns were predominant (Figures 3(E)-(G)). In contrast to the male pelvic floor, it has been shown that the VIP-dominant nerve supply is rich in the female pelvic floor, especially along the vagina $[49,50]$. However, VIP-positive fibers in the female pelvic floor may include sensory fibers [51,52].

\section{PERINEAL SMOOTH MUSCLES}

When observed in sagittal sections, the upper half of the rectovaginal septum, or Denonvillers' fascia in females, is so thin or weak as a target of surgical repair (Figure 5). 


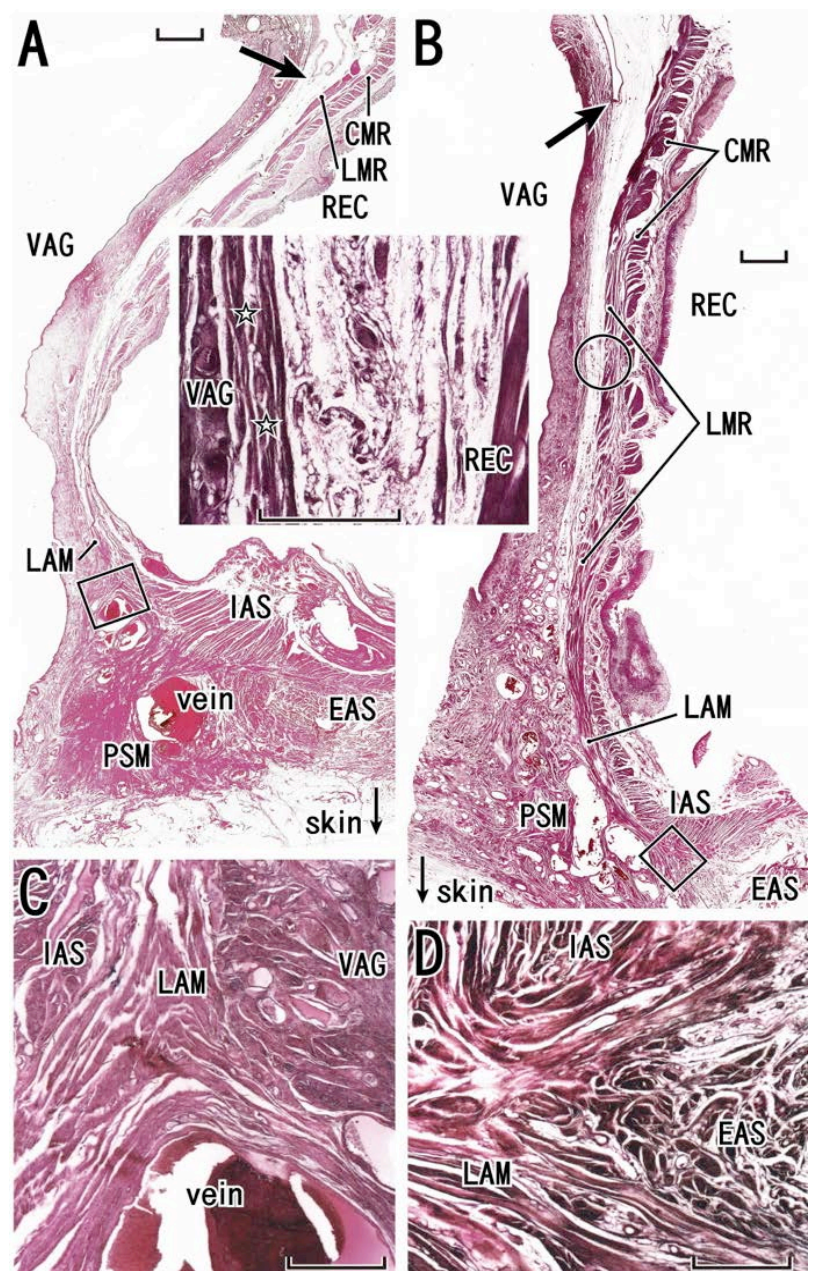

Figure 5. Entire views of the rectovaginal interface. Sagittal sections. HE staining. Panel A (a 78-year-old woman) and panel B (a 75-year-old woman) display region-specific difference in the rectovaginal interface (scale bar, $10 \mathrm{~mm}$ ). Arrows indicate the bottom of the peritoneal cavity. The rectovaginal septum is unclear especially in the superior part in both specimens: a higher magnification view of a circle in panel B is inserted between panels A and B. Stars in the insert are most likely to indicate the vaginal adventitia rather than the rectovaginal septum. The longitudinal muscle layer of the rectum (LMR) is continuous with the longitudinal anal muscle (LAM) without a contribution of the endopelvic fascia (see Figures $1(\mathrm{~A})$ and $(\mathrm{C})$ ) and the longitudinal muscle reaches perineal smooth muscles (PSM) in panel A or the external anal sphincter (EAS) in panel B. Panel C (or D), corresponding to a square in panel A (or B), exhibits the inferiormost part of the longitudinal anal muscle (scale bar, $1 \mathrm{~mm}$ ). CMR, circular muscle layer of the rectum (smooth muscles); IAS, internal anal sphincter; REC, rectum; VAG, vagina.

Inferiorly, the septum reaches the bilateral large masses of the perineal smooth muscles (PSMs) occupying a large subcutaneous space on the posterolateral and lateral sides of the distal vagina and vestibulum (Figures 5-10). Because their major interest was individual variations in perineal body morphology, Soga et al. [5] called PSMs
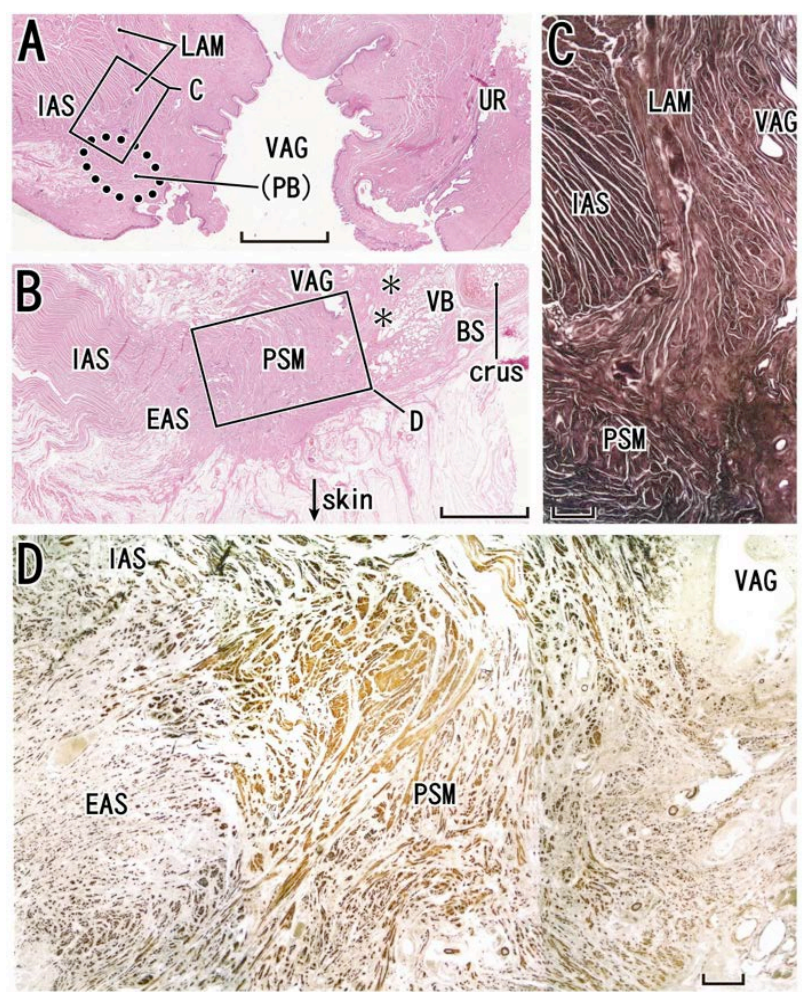

Figure 6. Perineal smooth muscles in sagittal sections from an 82-year-old woman. Panels A-C, HE staining. Panel A, at the almost midsagittal plane, displays the longitudinal anal muscle (LAM) running inferiorly (scale bar, $10 \mathrm{~mm}$ ), while panel B, 15 $\mathrm{mm}$ lateral to panel A, exhibits a bulky mass of perineal smooth muscles (PSM). The definite perineal body is not seen (PB with parenthesis in panel A). Asterisks in panel B indicate a candidate of the perineal membrane. Panel C (scale bar, $1 \mathrm{~mm}$ ) is a higher magnification view of a square in panel A. Panel D (immunohistochemistry for smooth muscles; scale bar, $1 \mathrm{~mm}$ ), corresponding to a square in panel $\mathrm{B}$, shows irregularly arrayed smooth muscle fibers (perineal smooth muscles or PSM) those provide anterior insertions to the external and internal anal sphincters (EAS, IAS). BS, bulbospongiosus muscle; crus, crus clitoris; UR, urethra; VAG, vagina; VB, vestibular bulb.

the "lateral extension of the perineal body". In fact, the bilateral masses of the PSMs are connected by the perineal body (Figure 8), and together they are arranged in a catamaran-like configuration (i.e. a double-hulled boat). In fact, DeLancey [53] considered the perineal body and membrane to be critical factors for level III support. However, rather than the PSMs, the female perineal body is difficult to demonstrate because of variations in histology: it can be a round mass of irregularly arrayed smooth muscles (Figures 7(A) and 8(A)), a fatty tissue mass (Figure 9(A)), or part of the PSMs without a clear demarcation (Figure 6(A)). Nevertheless, irrespective of morphology, the perineal body as well as the PSMs receive the LAM at the inferior end (Figures 6(B), (C), 7(A) and 9(A)). Similarly to the perineal body [33,54], the PSMs appear to originated and migrate from the de- 


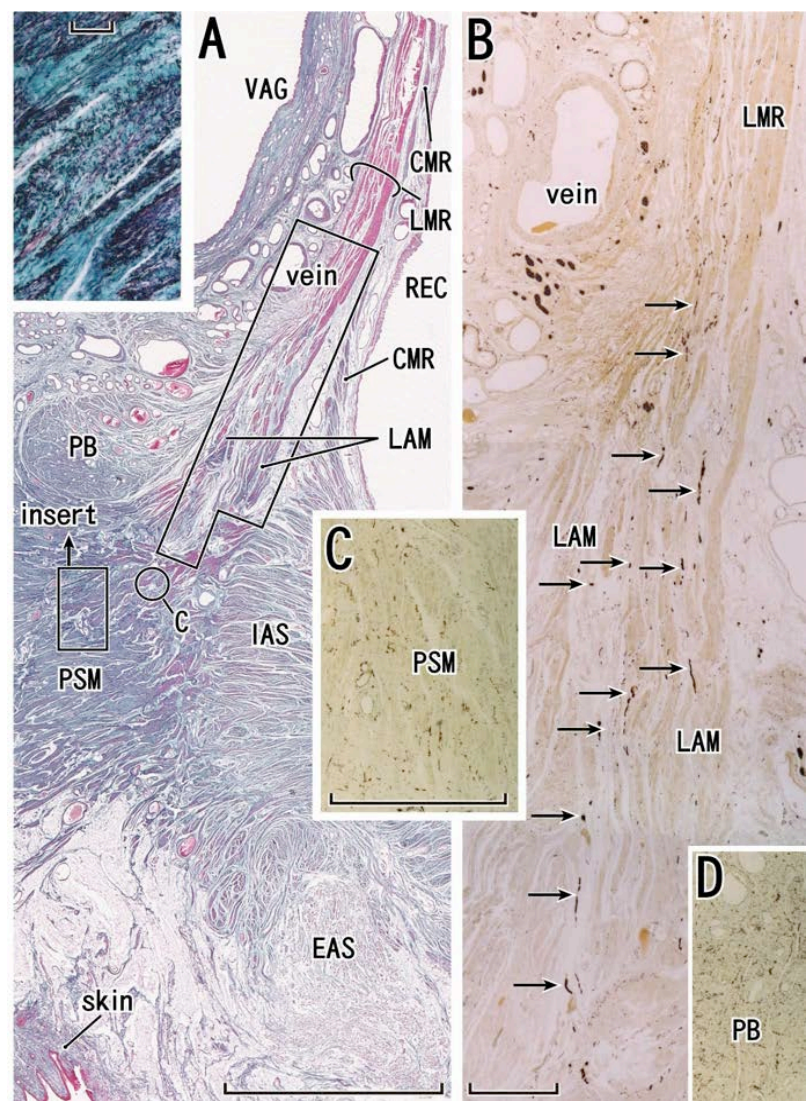

Figure 7. Longitudinal anal muscle and perineal smooth muscles at the almost mid-sagittal plane in a specimen from a 78year-old woman. The same specimen as shown Fig. 1A. Panel A shows elastica-Masson staining (striated muscles, red; collagen fibers, green; elastic fibers, black or dark blue). The longitudinal muscle layer of the rectum (LMR) runs inferiorly, continues to the longitudinal anal muscle (LAM) and insert into perineal smooth muscles (PSM) without a contribution of smooth muscle fibers from the endopelvic fascia (see Figures $1(A)$ and $(\mathrm{C})$ ). A round mass (PB), comprising of smooth muscles and elastic fibers, is a most likely candidate of the perineal body. Insert in panel A displays a higher magnification view of a small square in panel A: perineal smooth muscles contain abundant elastic fibers (black wavy lines). Panel B, corresponding to a long square in panel A, exhibits immunohistochemistry for all nerves. The longitudinal anal muscle contains a series of descending nerves (arrows in panel B). Panel C, corresponding to a circle in panel A, displays abundant nerve terminals in perneal smooth muscles. Panel D (at the magnification same as in panel C) exhibits immunohistochemistry for all nerves in a small part of the candidate of the perineal body. The nerve terminal distribution is denser than perineal smooth muscles. Scale bars: $10 \mathrm{~mm}$ in panel A; $1 \mathrm{~mm}$ in panels B and C; $0.1 \mathrm{~mm}$ in the insert in panel A. CMR, circular muscle layer of the rectum; EAS and IAS, external and internal anal sphincters; REC, rectum; VAG, vagina.

veloping longitudinal muscle layer of the fetal rectum.

At the rectovaginal interface, the longitudinal smooth muscle layer of the rectum continues to the LAM without any contribution by the endopelvic fascia (Figures 5(A),

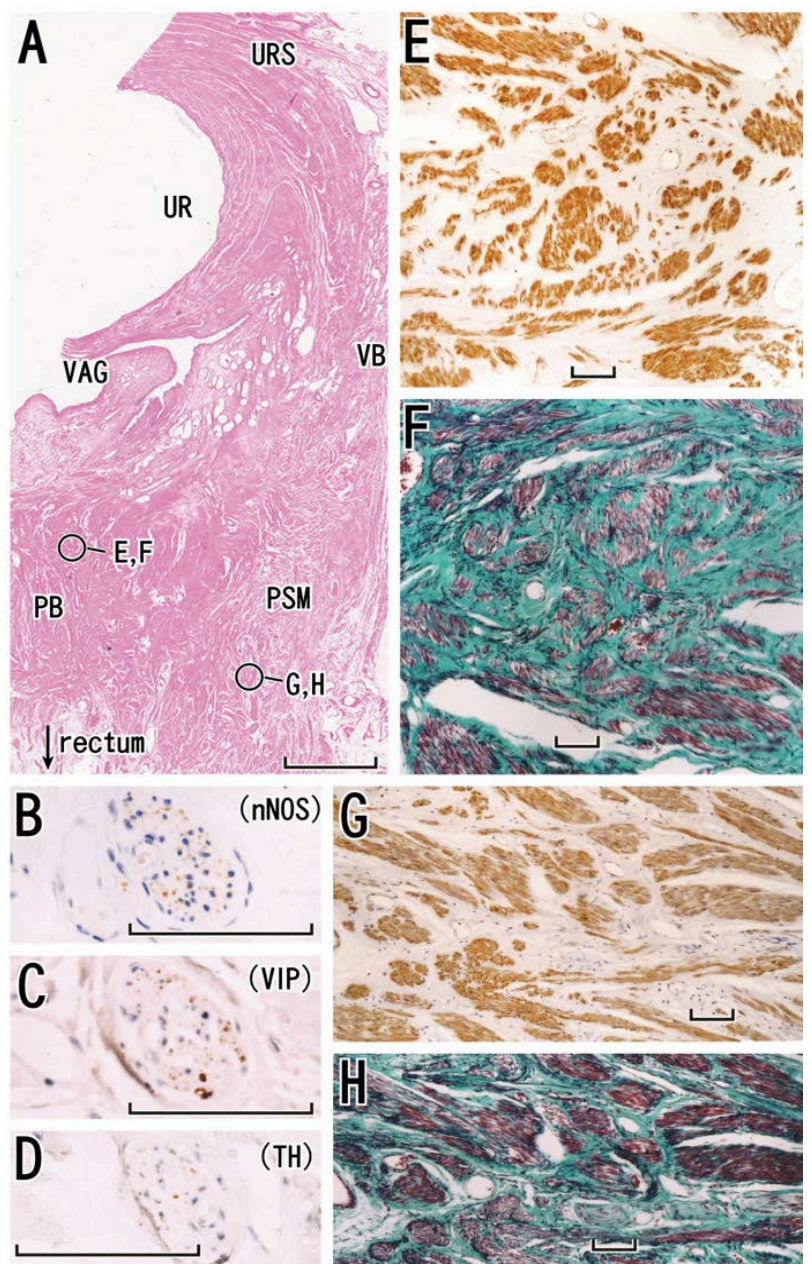

Figure 8. Perineal smooth muscles and the perineal body in a horizontal section of a specimen from a 79-year-old woman. The perineal body (PB) is evident in panel A. The urethra (UR) is highly dilated due to long-term catheterization. Perineal smooth muscle (PSM) occupied in a large area in the lateral side of the perineal body. A nerve in perineal smooth muscles is shown in panel B (immunohistochemistry for parasympathetic nerve fibers; neuronal nitric oxide synthase or nNOS), panel C (immunohistochemistry for parasympathetic nerve fibers; vasoactive intestinal polypeptide or VIP) and panel D (immunohistochemistry for sympathetic nerve fibers). Panel E (immunohistochemistry for smooth muscles) and panel F (elastica-Masson staining; collagen fibers, green; elastic fibers, black) are higher magnification views of a circle in the perineal body. Likewise, panel G (immunohistochemistry for smooth muscles) and panel $\mathrm{H}$ (elastica-Masson staining) exhibit a circle in perineal smooth muscles at the higher magnification. Scale bars: 10 $\mathrm{mm}$ in panel A; $0.1 \mathrm{~mm}$ in panels B-H. URS, urethral rhabdosphincter; VAG, vagina; VB, vestibular bulb.

(B) and 7(A)). Thus, on the anterior side of the lower rectum, there is no "conjoint muscle coat of the rectum" in the true sense (see the subsection "Levator ani muscle, collagenous fibers and elastic fibers"). However, irrespective of whether joining of the endopelvic fascia is present or absent, some researchers may consider the inferior 

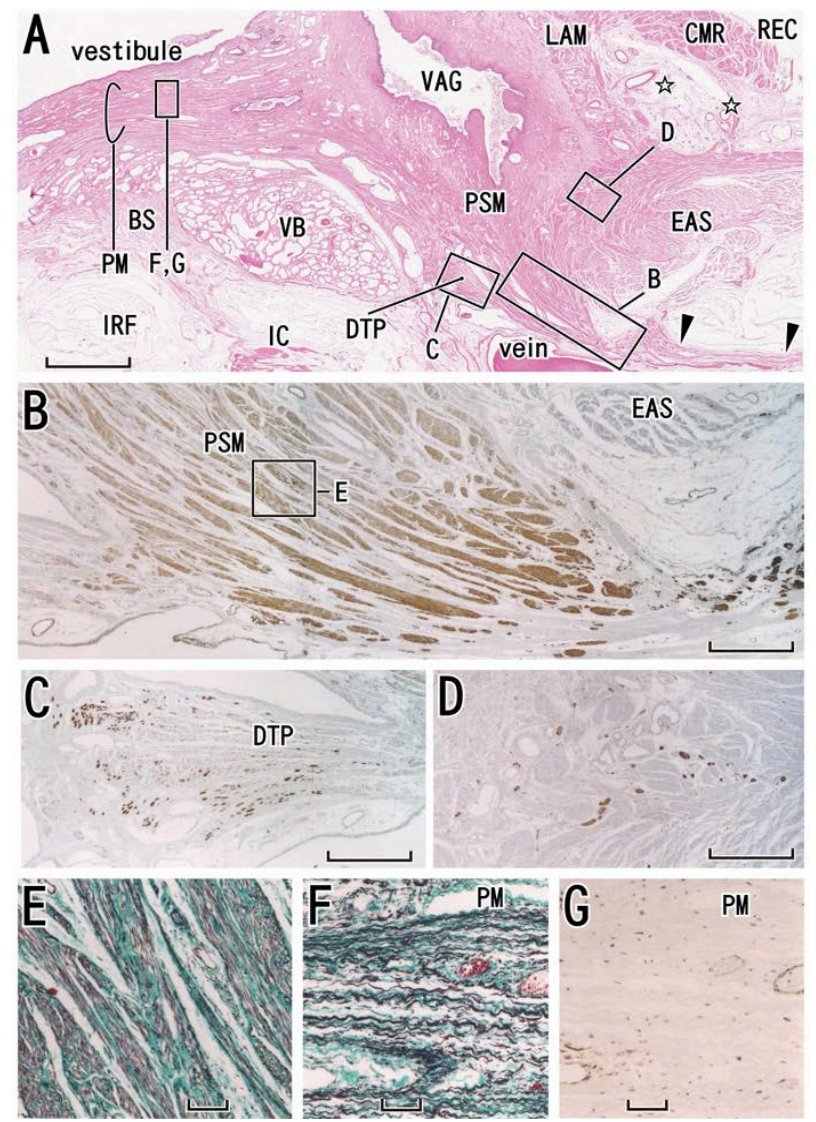

Figure 9. Perineal smooth muscles provide a posterior attachment to the perineal membrane. Almost horizontal sections of a specimen from a 76-year-old woman. In panel A (HE staining), the vagina (VAG) and rectum (REC) are not tightly attached but separated by a relatively loose tissue (stars). Perineal smooth muscles (PSM) connect between the external anal sphincter (EAS) and vagina and the former muscles also provide a posterior attachment to the perineal membrane (PM). The membrane extends anteriorly along the medial or vestibular side of the vestibular bulb (VB). A thick fascia extending posteriorly (arrowheads) appears to have no name other than "one of the skin ligaments". Panel B (immunohistochemistry for smooth muscles), corresponding to a long square with B in panel A, shows a higher magnification view of perineal smooth muscles. Panel C (immunohistochemistry of desmin showing striated muscle fiber ends) displays the vestigial deep transverse perineal muscle (DTP). Panel D (immunohistochemistry for all nerves), corresponding to a square with $\mathrm{D}$ in panel $\mathrm{A}$, exhibits nerves in perineal smooth muscles. Panel E (elastica-Masson staining), corresponding to a square in panel B, shows abundant elastic fibers (black) in the perineal smooth muscles. The perineal membrane (PM) is composed of elastic fibers (panel E; elastica-Masson staining) but it contains no smooth muscles (panel G; immunohistochemistry for smooth muscles). Scale bars: $10 \mathrm{~mm}$ in panel A; $1 \mathrm{~mm}$ in panels B-D; $0.1 \mathrm{~mm}$ in panel E-G. BS, bulbospongiosus muscle; CMR, circular muscle layer of the rectum; IRF, ischiorectal fossa; IC, ischiocavernosus muscle; LAM, longitudinal anal muscle.

continuation of the longitudinal muscle layer of the rectum to be the LAM. The LAM ends at the PSMs, and the
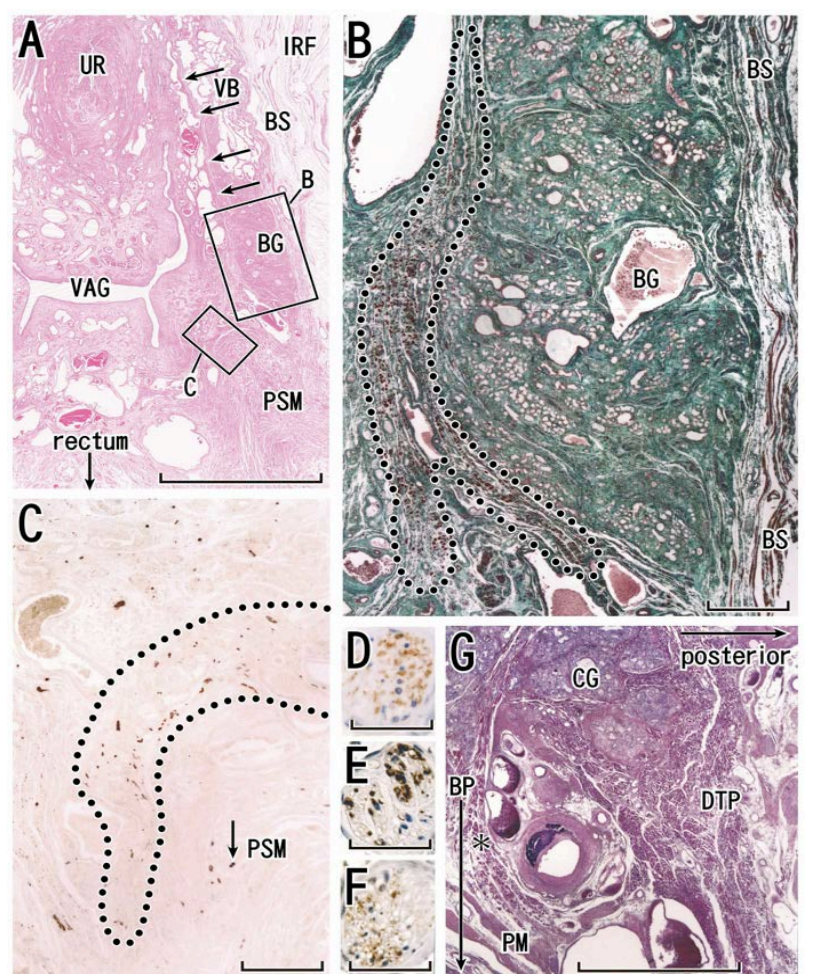

Figure 10. Deep transverse perineal muscle in females. Almost horizontal sections of a specimen from an 83-year-old woman (panels A-C). Panel A (HE staining) includes the urethra (UR), vagina (VAG), vestibular bulb (VB), Bartholin's gland (BG) and external anal sphincter (EAS). Note abundant veins around the distal vagina. Arrows in panel A indicate a candidate of the perineal membrane. Panel B, corresponding to a square with B in panel A, displays elastica-Masson staining (striated muscles, red; collagen fibers, green; elastic fibers, black) for easy identification of striated muscle fibers. In contrast to longitudinal muscle fibers of the bulbospongiosus muscle (BS), cross sections of another striated muscle (a candidate of the deep transverse perineal muscle in females; encircled by dotted line) occupies in the immediately medial side of Bartholin's gland). Panel C (immunohistochemistry for all nerves) exhibits a group of nerves (encircled by dotted line) running along the anterior margin of perineal smooth muscles (PSM). Panel D (immunohistochemistry for nitric oxide synthase or nNOS), panel E (immunohistochemistry for vasoactive intestinal polypeptide or VIP) and panel F (immunohistochemistry for tyrosine hydroxylase or $\mathrm{TH}$ ) demonstrate transmitters in a thin nerve indicated by arrow in panel C. Panels D-F are near sections of panel C. Panel G shows a sagittal section of a male specimen (an 82-year-old man) including the typical morphology of the deep transverse perineal muscle (DTP) that is attached to Cowper's gland (CG). In view of topographical relation with a gland, the female striated muscle shown in panel B is similar to the male transverse perineal muscle. Asterisk in panel D indicates an aberrant bundle of the transverse muscle. Scale bars: $10 \mathrm{~mm}$ in panel A; $1 \mathrm{~mm}$ in panels $\mathrm{B}, \mathrm{C}$ and $\mathrm{G} ; 0.05 \mathrm{~mm}$ in panels $\mathrm{D}, \mathrm{E}$ and F. BP, bulbus penis (male); IRF, ischiorectal fossa; PM, perineal membrane (male).

same PSMs provide the major anterior insertion to the external anal sphincter (Figures 6(B), 7(A) and 9(A)). 
Conversely, the strong striated sphincter seems to play a dynamic role in supporting the rectovaginal interface to maintain a suitable antero-posterior length of the urogenital hiatus against intra-abdominal pressure. The anterior attachment of the anal sphincter formed by the PSMs seems to be adapted more effectively to mechanical stress than the static posterior attachment formed by elastic fibers, i.e., the anococcygeal ligament and raphe [55].

At its posterior end, the female perineal membrane ends at the PSMs (Figure 9(A)). The typical multilayered perineal membrane is rarely found in elderly Japanese women [8] (Figures $9(\mathrm{~F})$ and $(\mathrm{G})$ ) because the PSMs have enlarged, destroying the membrane from the superficial and posterior sides. Sometimes, most parts of the perineal membrane appear to be integrated into the bulky PSMs. Accordingly, the mass of the PSMs occupies the center of the fibrous skeleton in the female perineum, and integrates both a group of vertical structures (vaginal wall smooth muscles and the LAM) and a group of structures extending horizontally and/or frontally (the perineal membrane and perineal body). We propose a schematic representation showing the critical position of the PSMs (Figure 11; see the final paragraph of this subsection). In the vertical structures, the urethra is tightly connected with the vagina $[56,57]$ because of vaginal descent with marked reconstruction of the urethral posterior wall [58]. The female urethral smooth muscles are characterized by their thick longitudinal layer[59]. The PSMs in females correspond to the recto-urethralis muscle in males: the male LAM also insert into the recto-urethralis muscle $[60,61]$. However, in contrast to the smooth muscle mass in males, PSMs are unlikely to contain the cavernous nerves in females.

As typically seen in arterial walls, smooth muscles and elastic fibers usually coexist because elastic fibers are necessary for maintaining the 3-dimesnional configuration of smooth muscle fibers [62]. As well as the vascular wall, in the pelvic floor, smooth muscle and elastic fibers often coexist (Figures 1(C), (D), 3(B), (C), 8(E)(H) and 9(B), (E)). Do fibers of PSMs exert synchronized action with the levator ani under the control of nerve impulses? Smooth muscles in the pelvic floor connective tissue are not arrayed regularly and are directed at random (Figures 6(D) and 8(E)-(H)). PSMs contain abundant nerve terminals (Figures 7(C), (D) and $10(C)$ ), but their density is much lower than in the circular and longitudinal smooth muscle layers of the rectum (Figure 1(B)). The PSMs are likely to differ in function from the regularly arrayed smooth muscle structures typically seen in the intestine. The latter type of structure shows organized contraction or peristalsis under the control of nerves and hormones, but smooth muscles in connective tissue may not, in view of their random arrangement. This is somewhat reminiscent of the nature of smooth muscle cells or fibers in the walls of arteries, which can act against blood pressure without nerve or hormonal control (i.e., Bayliss' rule [63,64]).

Connective tissue composed of smooth muscle would seem to function as an ideal barrier, septum or protector against mechanical stress because, even without innervation, smooth muscle fibers resist (not absorb) pressure in accordance with Bayliss' rule. This function seems to be much stronger than the passive action of elastic fibers. However, at the full term of pregnancy, PSMs are most likely to become highly relaxed under various hormonal influences to assist delivery. In contrast, extracellular collagenous and elastic fibers would be torn or elongated by excess tension during vaginal delivery. In addition, the venous plexus located around the vagina is well developed in multiparous women $[5,42]$. The venous plexus also appears to have a role in relaxation of the rectovaginal interface during delivery. Nevertheless, any dynamic function of structures other than PSMs would appear to be unlikely.

The "integrated pelvic floor theory" [65] attributes a key role to the LAM in the static and dynamic support of the pelvic viscera, being involved in the closure and opening of the urethra and anal canal. Petros' system resembles the line drawings published by Courtney [66], and according to both of them, the levator ani gives off "striated" muscle fibers extending longitudinally and inferiorly along the rectum. However, except for Macchi et al. [22], no other researchers have supported the existence of such striated muscles (e.g., Arakawa et al. [20]). Because Courtney [66] based his conclusions on gross anatomy and because the histological demonstration by Petros [65] was limited to the pubo-urethral ligament, their overemphasis of the morphology of the levator ani seemed to result from a lack of histological observations. We agree that, in the female pelvic floor, there is a complex of smooth muscles, especially vertically running muscles, including the LAM. However, smooth muscles are unlikely to play an active traction role without the cooperation of striated muscles. Moreover, the real LAM is located distantly from the female urethra or its rhabdosphincter, but it ends more posteriorly around the vagina and vaginal vestibule [13]. In accordance with Bayliss' rule, rather than traction or elevation, the PSMs seem to act as an ideal barrier, septum or protector against mechanical stress such as that resulting from intra-abdominal pressure. In addition, Petros's "posterior levator plate" (the anococcygeal ligament and raphe) is elastic $[55,67]$ and unlikely to provide any strong posterior traction force. Another review [68] has focused on the limited effect of the levator ani on urethral function in both genders. Consequently, to demonstrate the key role of the perineal smooth muscles and perineal body for integrated function of the LAM, anal sphincters and 
perineal membrane, we have proposed our "catamaran" model (Figure 11).

\section{DEEP TRANSVERSE PERINEL MEMBRANE AND THE UROGENITAL DIAPHRAGM}

Nakajima et al. [69] reported that the deep transverse perineal muscle is attached to Cowper's gland in males (Figure 10(G)), and is continuous with the urethral rhabdosphincter. With regard to the situation in females, Fritsch et al. [70] reported that the muscle was absent. Despite his excellent diagrams, which included the mem-

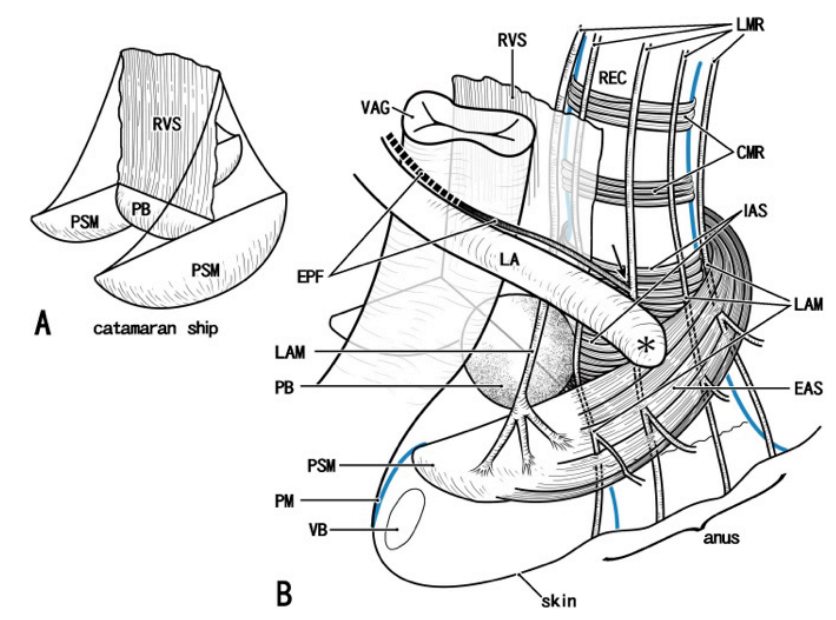

Figure 11. A catamaran ship model for a smooth musclemade fibrous skeleton in the female perineum. Panel A displays a key structure like a catamaran ship (a ship with a pair of bellies): the bellies correspond to a pair of bulky masses of perineal smooth muscles (PSM) and bilateral masses are connecting by the perineal body (PB) in the center. The sail corresponds to the rectovaginal septum (RVS). In panel B, we try to insert the vagina (VAG) and rectum (REC) in the key structure. The rectovaginal septum is often thin, fragmented or absent. Along the lateral and posterolateral aspects of the lower rectum (arrow), the longitudinal muscle layer of the rectum (smooth muscle; LMR) join smooth muscles from the endopelvic fascia (EPF) and they together provide the longitudinal anal muscle (LAM). However, along the anterior aspect of the lower rectum, the longitudinal muscle layer of the rectum simply continues to the longitudinal anal muscle without a contribution of the endopelvic fascia. At the rectovaginal interface, the longitudinal anal muscle inserts into perineal smooth muscles (PSM). In the lateral and posterolateral sides of the rectum, major parts of the longitudinal anal muscle ran through the intersphincteric space between the external and internal anal sphincters (EAS, IAS) to reach the anal skin, while minor parts penetrate the external sphincter. The perineal membrane (PM) extends anteriorly from the perineal smooth muscles, surrounds the anterolateral aspects of the vestibule. Due to our poor skill, we were not able to draw precisely the attachment (asterisk) of the levator ani muscle (LA) to the external sphincter. CMR, circular muscle layer of the rectum (smooth muscles); REC, rectum; VB, vestibular bulb. branous structure at the external genitalia, Oelrich [4,71] had a negative, rather than positive, opinion, because he had no opportunity to observe striated muscles in the membranous structures of the female. Oh and Kark [72] successfully demonstrated the transverse muscle in the lateral side of the perineal body in females, but their findings were not subsequently cited by others. We believe that the muscle is present, although vestigial, adjacent to Bartholin's gland (Figure 10(B)). Thus, in both genders, the muscle is located inferiorly or superficially relative to the perineal membrane, and adjacent to the major perineal gland. Nakajima et al. [69] considered that, because the deep transverse perineal muscle is not sheet-like but a 3-dimensional pillar continuous with the rhabdosphincter, previous researchers had found it difficult to identify, especially in histology preparations.

On the basis of magnetic resonance imaging studies, the strictest argument against the existence of the urogenital diaphragm was provided by Myers [73], who established a safe treatment for the retropubic veins in radical prostatectomy. He stated that "there is not a hint of what might be called Henle's artifact, his diaphragma urogenitale." Likewise, Mirilas and Skanadalakis [74] had a strongly negative opinion regarding the existence of the diaphragm. The deep transverse perineal muscle has long been considered the core of the urogenital diaphragm. However, in our cadaver dissections, when we approached the urogenital hiatus from the ischiorectal fossa lateral to the rectum and extending to the lateral side of the urethra or vagina, we were able to palpate a diaphragm-like structure containing 1) the urethral and urethrovaginal sphincters, 2) the mass of the PSMs, 3) the perineal membrane, 4) Bartholin's gland and its associated striated muscle fibers (the vestigial deep transverse perineal muscle) and/or 5) the vestibular bulb and its associated bulbospongiosus muscle. Likewise, for measurement of thickness using clinical imaging, Betschart et al. [75] considered the perineal membrane as a mass that includes striated muscle, smooth muscle and connective tissues. Because such a gross entity including multiple structures is a useful descriptor, we do not rule out the concept of the urogenital diaphragm.

\section{CONCLUDING REMARKS}

Usually in the body, not only muscle contraction force for action but also passive mechanical stress can be transferred through a series of collagenous tissues of different types. However, in the pelvic floor, both are passed from collagen fibers to an elastic fiber-smooth muscle complex. When they are coexisted, elastic fibers bundle and connect with smooth muscles to maintain the configuration of the latter. PSMs provide the major anterior insertion to the external anal sphincter and, con- 
versely, the strong striated sphincter seems to play a dynamic role in supporting the rectovaginal interface to maintain the antero-posterior length of the urogenital hiatus against prolapse. The bilateral large mass of the PSMs constitutes a pair of bases or cores of a smoothmuscle skeleton that includes both a group of vertical structures (the vaginal wall smooth muscles and the LAM) and a group of structures extending horizontally and/or frontally (the perineal membrane and perineal body). Randomly arrayed smooth muscle fibers in the PSMs are likely to act as a barrier, septum or protector against mechanical stress because, even without innervation, they would resist pressure in accordance with Bayliss' rule.

\section{ACKNOWLEDGEMENTS}

We are grateful to the individuals who donated their bodies after death to Tokyo Dental College for research and education on human anatomy without any socio-economic benefit. We also thank their families for agreeing to the donation, as well as their patience in waiting for return of their remains after study.

\section{REFERENCES}

[1] DeLancey, J.O. and Starr, R.A. (1990) Histology of the connection between the vagina and levator ani muscles. Implications for urinary tract function. The Journal of Reproductive Medicine, 35, 765-771.

[2] De Lancey, J.O. (1992) Anatomic aspects of vaginal eversion after hysterectomy. American Journal of Obstetrics \& Gynecology, 166, 1717-1728.

[3] DeLancey, J.O. (1993) Anatomy and biomechanics of genital prolapse. Clinical Obstetrics and Gynecology, 36, 897-909.

http://dx.doi.org/10.1097/00003081-199312000-00015

[4] Oelrich, T.M. (1983) The striated urogenital sphincter muscle in the female. Anatomy \& Physiology, 205, 223232. http://dx.doi.org/10.1002/ar.1092050213

[5] Soga, H., Nagata, I., Murakami, G., Yajima, T., Takenaka, A., Fujisawa, M. and Koyama, M. (2007) A histotopographic study of the perineal body in elderly women: The surgical applicability of novel histological findings. International Urogynecology Journal and Pelvic Floor Dysfunction, 18, 1423-1430. http://dx.doi.org/10.1007/s00192-007-0380-2

[6] Hudson, C.N., Sohaib, S.A., Shulver, H.M. and Reznek, R.H. (2002) The anatomy of the perineal membrane: Its relationship to injury in childbirth and episiotomy. Australian and New Zealand Journal of Obstetrics and Gynaecology, 42, 193-196. http://dx.doi.org/10.1111/j.0004-8666.2002.00193.x

[7] Stein, T.A. and DeLancey, J.O. (2008) Structure of the perineal membrane in females: Gross and microscopic anatomy. Obstetrics \& Gynecology, 111, 686-693. http://dx.doi.org/10.1097/AOG.0b013e318163a9a5

[8] Kato, M., Matsubara, A., Murakami, G., Abe, S., Ide, Y.,
Sato, I. and Usui, T. (2008) Female perineal membrane: A study using pelvic floor semiserial sections from elderly nulliparous and multiparous women. International Urogynecology Journal and Pelvic Floor Dysfunction, 19, 1663-1670. http://dx.doi.org/10.1007/s00192-008-0701-0

[9] Hinata, N., Murakami, G., Abe, S., Shibata, S., Morizane, S., Honda, M., Isoyama, T., Sejima, T. and Takenaka, A. (2013) Coexistence of elastic fibers with hyaluronic acid in the human urethral sphincter complex: A histological study. Journal of Urology, 190, 1313-1319. http://dx.doi.org/10.1016/j.juro.2013.04.023

[10] Donker, P.J. (1986) A study of the myelinated fibres in the branches of the pelvic plexus. Neurourology and Urodynamics, 5, 185-202. http://dx.doi.org/10.1002/nau.1930050207

[11] Ball Jr., T.P., Teichman, J.M., Sharkey, F.E., Rogenes, V.J. and Adrian Jr., E.K. (1997) Terminal nerve distribution to the urethra and bladder neck: Considerations in the management of stress urinary incontinence. Journal of Urology, 158, 827-829.

http://dx.doi.org/10.1016/S0022-5347(01)64329-5

[12] Niikura, H., Katahira, A., Utsunomiya, H., Takano, T., Ito, K., Nagase, S., Yoshinaga, K., Tokunaga, H., Toyoshima, M., Kinugasa, Y., Uchiyama, E., Murakami, G., Yabuki, Y. and Yaegashi, N. (2007) Surgical anatomy of intrapelvic fasciae and vesico-uterine ligament in nervesparing radical hysterectomy with fresh cadaver dissections. Tohoku Journal of Experimental Medicine, 212, 403-413. http://dx.doi.org/10.1620/tjem.212.403

[13] Kinugasa, Y., Arakawa, T., Abe, H., Rodriguez-Vazquez, J.F., Murakami, G. and Sugihara, K. (2013) Female longitudinal anal muscles or conjoint longitudinal coats extend into the subcutaneous tissue along the vaginal vestibule: A histological study using human fetuses. Yonsei Medical Journal, 54, 778-784.

http://dx.doi.org/10.3349/ymj.2013.54.3.778

[14] Trotter, J.A. (1993) Functional morphology of force transmission in skeletal muscle. A brief review. Acta Anatomica (Basel), 146, 205-222. http://dx.doi.org/10.1159/000147459

[15] Huijing, P.A. (1999) Muscle as a collagen fiber reinforced composite: a review of force transmission in muscle and whole limb. Journal of Biomechanics, 32, 329345. http://dx.doi.org/10.1016/S0021-9290(98)00186-9

[16] Purslow, P.P. (2002) The structure and functional significance of variations in the connective tissue within muscle. Comparative Biochemistry and Physiology Part A: Molecular \& Integrative Physiology, 133, 947-966. http://dx.doi.org/10.1016/S1095-6433(02)00141-1

[17] Osanai, H., Rodriguez-Vazquez, J.F., Abe, H., Murakami, G., Ohguro, H. and Fujimiya, M. (2011) Fetal check ligament connected between the conjunctiva and the medial and lateral recti. Investigative Ophthalmology \& Visual Science, 52, 7175-7179. http://dx.doi.org/10.1167/iovs.11-7426

[18] Benjamin, M., Toumi, H., Ralphs, J.R., Bydder, G., Best, T.M. and Milz, S. (2006) Where tendons and ligaments meet bone: Attachment sites ('enthuses') in relation to exercise and/or mechanical load. Journal of Anatomy, 
208, 471-490.

http://dx.doi.org/10.1111/j.1469-7580.2006.00540.x

[19] Williams, P.L. (1995) Gray’s anatomy. Churchill Livingstone, New York.

[20] Arakawa, T., Murakami, G., Nakajima, F., Matsubara, A., Ohtsuka, A., Goto, T. and Teramoto, T. (2004) Morphologies of the interfaces between the levator ani muscle and pelvic viscera, with special reference to muscle insertion into the anorectum in elderly Japanese. Anatomical Science International, 79, 72-81.

http://dx.doi.org/10.1111/j.1447-073x.2004.00069.x

[21] Hieda, K., Cho, K.H., Arakawa, T., Fujimiya, M., Murakami, G. and Matsubara, A. (2013) Nerves in the intersphincteric space of the human anal canal with special reference to their continuation to the enteric nerve plexus of the rectum. Clinical Anatomy, 26, 843-854. http://dx.doi.org/10.1002/ca.22227

[22] Macchi, V., Porzionato, A., Stecco, C., Vigato, E., Parenti, A. and De Caro, R. (2008) Histo-topographic study of the longitudinal anal muscle. Clinical Anatomy, 21, 447452. http://dx.doi.org/10.1002/ca.20633

[23] Kannus, P. (2000) Structure of the tendon connective tissue. Scandinavian Journal of Medicine \& Science in Sports, 10, 312-320.

http://dx.doi.org/10.1034/j.1600-0838.2000.010006312.x

[24] Ritty, T.M., Ditsios, K. and Starcher, B.C. (2002) Distribution of the elastic fiber and associated proteins in flexor tendon reflects function. The Anatomical Record, 268, 430-440. http://dx.doi.org/10.1002/ar.10175

[25] Stecco, C., Porzionato, A., Macchi, V., Tiengo, C., Parenti, A., Aldegheri, R., Delmas, V. and De Caro, R. (2006) Histological characteristics of the deep fascia of the upper limb. Italian Journal of Anatomy and Embryology, 111, 105-110.

[26] Ochala, J., Frontera, W.R., Dorer, D.J., Van Hoecke, J. and Krivickas, L.S. (2007) Single skeletal muscle fiber elastic and contractile characteristics in young and older men. Journals of Gerontology Series A: Biological Sciences and Medical Sciences, 62, 375-381. http://dx.doi.org/10.1093/gerona/62.4.375

[27] Kono, R., Poukens, V. and Demer, J.L. (2002) Quantitative analysis of the structure of the human extraocular muscle pulley system. Investigative Ophthalmology \& Visual Science, 43, 2923-2932.

[28] Miller, J.L., Watkin, K.L. and Chen, M.F. (2002) Muscle, adipose, and connective tissue variations in intrinsic musculature of the adult human tongue. Journal of Speech Language and Hearing Research, 45, 51-65. http://dx.doi.org/10.1044/1092-4388(2002/004)

[29] Murakami, G., Nakajima, F., Sato, T.J., Tsugane, M.H., Taguchi, K. and Tsukamoto, T. (2002) Individual variations in aging of the male urethral rhabdosphincter in Japanese. Clinical Anatomy, 15, 241-252. http://dx.doi.org/10.1002/ca.10015

[30] Hahn, M.S., Kobler, J.B., Starcher, B.C., Zeitels, S.M. and Langer, R. (2006) Quantitative and comparative studies of the vocal fold extracellular matrix. I: Elastic fibers and hyaluronic acid. Annals of Otology, Rhinology, and
Laryngology, 115, 156-164.

[31] Korn, G.P., Martins, J.R., Park, S.W., Mendes, A., Kobayashi, E.Y., Nader, H.B. and De Biase, N.G. (2011) Concentration of hyaluronic acid in human vocal folds in young and old subjects. Otolaryngology_Head and Neck Surgery, 145, 981-986.

http://dx.doi.org/10.1177/0194599811419457

[32] Kawase, T., Shibata, S., Katori, Y., Ohtsuka, A., Murakami, G. and Fujimiya, M. (2012) Elastic fiber-mediated enthesis in the human middle ear. Journal of Anatomy, 221, 331-340. http://dx.doi.org/10.1111/j.1469-7580.2012.01542.x

[33] Hirata, E., Fujiwara, H., Hayashi, S., Ohtsuka, A., Abe, S., Murakami, G. and Kudo, Y. (2011) Intergender differences in histological architecture of the fascia pelvis parietalis: A cadaveric study. Clinical Anatomy, 24, 469477. http://dx.doi.org/10.1002/ca.21042

[34] Zhu, L., Lang, J., Feng, R., Chen, J. and Wong, F. (2004) Estrogen receptor in pelvic floor tissues in patients with stress urinary incontinence. International Urogynecology Journal and Pelvic Floor Dysfunction, 15, 340-343.

[35] Mauroy, B., Goullet, E., Stefaniak, X., Bonnal, J.L. and Amara, N. (2000) Tendinous arch of the pelvic fascia: Application to the technique of paravaginal colposuspension. Surgical and Radiologic Anatomy, 22, 73-79. http://dx.doi.org/10.1007/s00276-000-0073-8

[36] Delancey, J.O. (2002) Fascial and muscular abnormalities in women with urethral hypermobility and anterior vaginal wall prolapse. American Journal of Obstetrics and Gynecology, 187, 93-98.

[37] Pit, M.J., De Ruiter, M.C., Lycklama, A.N.A.A., Marani, E. and Zwartendijk, J. (2003) Anatomy of the arcus tendineus fasciae pelvis in females. Clinical Anatomy, 16, 131-137. http://dx.doi.org/10.1002/ca.10102

[38] Albright, T.S., Gehrich, A.P., Davis, G.D., Sabi, F.L. and Buller, J.L. (2005) Arcus tendineus fascia pelvis: A further understanding. American Journal of Obstetrics and Gynecology, 193, 677-681.

[39] Tamakawa, M., Murakami, G., Takashima, K., Kato, T. and Hareyama, M. (2003) Fascial structures and autonomic nerves in the female pelvis: A study using macroscopic slices and their corresponding histology. Anatomical Science International, 78, 228-242. http://dx.doi.org/10.1046/j.0022-7722.2003.00061.x

[40] Nagata, I., Murakami, G., Suzuki, D., Furuya, K., Koyama, M. and Ohtsuka, A. (2007) Histological features of the rectovaginal septum in elderly women and a proposal for posterior vaginal defect repair. International Urogynecology Journal, 18, 863-868.

http://dx.doi.org/10.1007/s00192-006-0249-9

[41] Takenaka, A., Hara, R., Soga, H., Murakami, G. and Fujisawa, M. (2005) A novel technique for approaching the endopelvic fascia in retropubic radical prostatectomy, based on an anatomical study of fixed and fresh cadavers. BJU International, 95, 766-771.

[42] Hirata, E., Koyama, M., Murakami, G., Ohtsuka, A., Abe, S., Ide, Y., Fujiwara, H. and Kudo, Y. (2011) Comparative histological study of levels 1-3 supportive tissues us- 
ing pelvic floor semiserial sections from elderly nulliparous and multiparous women. Journal of Obstetrics and Gynaecology Research, 37, 13-23. http://dx.doi.org/10.1111/j.1447-0756.2010.01298.x

[43] Zhai, L.D., Liu, J., Li, Y.S., Yuan, W. and He, L. (2009) Denonvilliers' fascia in women and its relationship with the fascia propria of the rectum examined by successive slices of celloidin-embedded pelvic viscera. Diseases of the Colon \& Rectum, 52, 1564-1571. http://dx.doi.org/10.1007/DCR.0b013e3181a8f75c

[44] Kato, M., Niikura, H., Yaegashi, N., Murakami, G., Tatsumi, H. and Matsubara, A. (2008) Histotopography of the female cavernous nerve: A study using donated fetuses and adult cadavers. International Urogynecology Journal, 19, 1687-1695. http://dx.doi.org/10.1007/s00192-008-0713-9

[45] Lindsey, I., Guy, R.J., Warren, B.F. and Mortensen, N.J. (2000) Anatomy of Denonvilliers' fascia and pelvic nerves, impotence, and implications for the colorectal surgeon. British Journal of Surgery, 87, 1288-1299.

[46] Kinugasa, Y., Murakami, G., Uchimoto, K., Takenaka, A., Yajima, T. and Sugihara, K. (2006) Operating behind Denonvilliers' fascia for reliable preservation of urogenital autonomic nerves in total mesorectal excision: A histologic study using cadaveric specimens, including a surgical experiment using fresh cadaveric models. Diseases of the Colon \& Rectum, 49, 1024-1032. http://dx.doi.org/10.1007/s10350-006-0557-7

[47] Zhang, C., Ding, Z.H., Li, G.X., Yu, J., Wang, Y.N. and Hu, Y.F. (2010) Perirectal fascia and spaces: Annular distribution pattern around the mesorectum. Diseases of the Colon \& Rectum, 53, 1315-1322. http://dx.doi.org/10.1007/DCR.0b013e3181e74525

[48] Hieda, K., Hinata, N., Sasaki, H., Morizane, S., Murakami, G., Abe, S. and Matsubara. A. (2014) Topohistology of sympathetic and parasympathetic nerve fibers in pelvic plexus branches: An immunohistochemical study using donated elderly cadavers. Anatomy \& Cell Biology, in Press.

[49] Hoyle, C.H., Stones, R.W., Robson, T., Whitley, K. and Burnstock, G. (1996) Innervation of vasculature and microvasculature of the human vagina by NOS and neuropeptide-containing nerves. Journal of Anatomy, 188, 633644.

[50] Hong, X., Huang, L. and Song, Y. (2008) Role of vasoactive intestinal peptide and pituitary adenylate cyclase activating polypeptide in the vaginal wall of women with stress urinary incontinence and pelvic organ prolapse. International Urogynecology Journal, 19, 1151-1157. http://dx.doi.org/10.1007/s00192-008-0585-z

[51] Maggi, C.A., Giuliani, S., Santicioli, P., Patacchini, R., Said, S.I., Theodorsson, E., Turini, D., Barbanti, G., Giachetti, A. and Meli, A. (1990) Direct evidence for the involvement of vasoactive intestinal polypeptide in the motor response of the human isolated ileum to capsaicin. European Journal of Pharmacology, 185, 169-178. http://dx.doi.org/10.1016/0014-2999(90)90637-L

[52] Jacobson, E.D., Berguer, R., Pawlik, W.W. and Hottenstein, O.D. (1994) Mesenteric purinergic and peptidergic vasodilators. Physiology of the Gastrointestinal Tract,
Raven, New York.

[53] De Lancey, J.O. (1999) Structural anatomy of the posterior pelvic compartment as it relates to rectocele. American Journal of Obstetrics \& Gynecology, 180, 815-823. http://dx.doi.org/10.1016/S0002-9378(99)70652-6

[54] Arakawa, T., Hayashi, S., Kinugasa, Y., Murakami, G. and Fujimiya, M. (2010) Development of the external anal sphincter with special reference to intergender difference: Observations of mid-term fetuses (15-30 weeks of gestation). Okajimas Folia Anatomica Japonica, 87, 49-58. http://dx.doi.org/10.2535/ofaj.87.49

[55] Kinugasa, Y., Arakawa, T., Abe, S., Ohtsuka, A., Suzuki, D., Murakami, G., Fujimiya, M. and Sugihara, K. (2011) Anatomical reevaluation of the anococcygeal ligament and its surgical relevance. Diseases of the Colon \& Rectum, 54, 232-237.

http://dx.doi.org/10.1007/DCR.0b013e318202388f

[56] DeLancey, J.O. (1994) Structural support of the urethra as it relates to stress urinary incontinence: The hammock hypothesis. American Journal of Obstetrics and Gynecology, 170, 1713-1720.

[57] Hinata, N., Murakami, G., Abe, S., Honda, M., Isoyama, T., Sejima, T. and Takenaka, A. (2012) Detailed histological investigation of the female urethra: Application to radical cystectomy. Journal of Urology, 187, 451-456. http://dx.doi.org/10.1016/j.juro.2011.10.037

[58] Masumoto, H., Rodriguez-Vazquez, J.F., Verdugo-Lopez, S., Murakami, G. and Matsubara, A. (2011) Fetal topographical anatomy of the female urethra and descending vagina: A histological study of the early human fetal urethra. Annals of Anatomy, 193, 500-508. http://dx.doi.org/10.1016/j.aanat.2011.03.010

[59] Kurihara, M., Murakami, G., Kajiwara, M., Taguchi, K., Tsukamoto, T. and Usui, T. (2004) Lack of the complete circular rhabdosphincter and a distinct circular smooth muscle layer around the proximal urethra in elderly Japanese women: An anatomical study. International Urogynecology Journal, 15, 85-94.

http://dx.doi.org/10.1007/s00192-003-1107-7

[60] Matsubara, A., Murakami, G., Arakawa, T., Yasumoto, H., Mutaguchi, K., Akita, K., Asano, K., Mita, K. and Usui, T. (2003) Topographic anatomy of the male perineal structures with special reference to perineal approaches for radical prostatectomy. International Journal of Urology, 10, 141-148. http://dx.doi.org/10.1046/j.1442-2042.2003.00585.x

[61] Uchimoto, K., Murakami, G., Kinugasa, Y., Arakawa, T., Matsubara, A. and Nakajima, Y. (2007) Rectourethralis muscle and pitfalls of anterior perineal dissection in abdominoperineal resection and intersphincteric resection for rectal cancer. Anatomical Science International, 82, 8-15. http://dx.doi.org/10.1111/j.1447-073X.2006.00161.x

[62] Dingemans, K.P., Teeling, P., Lagendijk, J.H. and Becker, A.E. (2000) Extracellular matrix of the human aortic media: An ultrastructural histochemical and immunohistochemical study of the adult aortic media. Anatomical Record, 258, 1-14.

http://dx.doi.org/10.1002/(SICI)1097-0185(20000101)25 8:1<1::AID-AR1>3.0.CO;2-7 
[63] Nelson, M.T. (1998) Bayliss, myogenic tone and volumeregulated chloride channels in arterial smooth muscle. Journal of Physiology, 507, 629. http://dx.doi.org/10.1111/j.1469-7793.1998.629bs.x

[64] Ji, G., Barsotti, R.J., Feldman, M.E. and Kotlikoff, M.I. (2002) Stretch-induced calcium release in smooth muscle. Journal of General Physiology, 119, 533-543. http://dx.doi.org/10.1085/jgp.20028514

[65] Petros, P. (2004) The female pelvic floor: Function, dysfunction and management according to the integral theory. Springer, Berlin. http://dx.doi.org/10.1007/978-3-662-05445-1

[66] Courtney, H. (1950) Anatomy of the pelvic diaphragm and anorectal musculature as related to sphincter preservation in anorectal surgery. American Journal of Surgery, 79, 155-173. http://dx.doi.org/10.1016/0002-9610(50)90208-X

[67] Kinugasa, Y., Arakawa, T., Abe, H., Abe, S., Cho, B.H., Murakami, G. and Sugihara, K. (2012) Anococcygeal raphe revisited: A histological study using mid-term human fetuses and elderly cadavers. Yonsei Medical Journal, 53, 849-855. http://dx.doi.org/10.3349/ymj.2012.53.4.849

[68] Hinata, N. and Murakami, G. (2014) The urethral rhabdosphincter, levator ani muscle and perineal membrane: A review. BioMed Research International, 2014.

[69] Nakajima, F., Takenaka, A., Uchiyama, E., Hata, F., Suzuki, D. and Murakami, G. (2007) Macroscopic and histotopographic study of the deep transverse perineal muscle (musculus transversus perinei profundus) in elderly Japanese. Annals of Anatomy, 189, 65-74.

http://dx.doi.org/10.1016/j.aanat.2006.06.014

[70] Fritsch, H., Lienemann, A., Brenner, E. and Ludwikowski, B. (2004) Clinical anatomy of the pelvic floor. Advances in anatomy, embryology and cell biology. Vol. 175, Springer, Berlin, pp. 1-64. http://dx.doi.org/10.1007/978-3-642-18548-9

[71] Oelrich, T.M. (1980) The urethral sphincter muscle in the male. American Journal of Anatomy, 158, 229-246. http://dx.doi.org/10.1002/aja.1001580211

[72] Oh, C. and Kark, A.E. (1973) Anatomy of the perineal body. Diseases of the Colon \& Rectum, 16, 444-454. http://dx.doi.org/10.1007/BF02588867

[73] Myers, R.P. (2001) Practical surgical anatomy for radical prostatectomy. Urologic Clinics of North America, 28, 473490. http://dx.doi.org/10.1016/S0094-0143(05)70156-7

[74] Mirilas, P. and Skandalakis, J.E. (2004) Urogenital diaphragm: An erroneous concept casting its shadow over the sphincter urethrae and deep perineal space. Journal of the American College of Surgeons, 198, 279-290. http://dx.doi.org/10.1016/j.jamcollsurg.2003.07.022

[75] Betschart, C., Scheiner, D., Maake, C., Vich, M., Slomianka, L., Fink, D. and Perucchini, D. (2008) Histomorphological analysis of the urogenital diaphragm in elderly women: A cadaver study. International Urogynecology Journal, 19, 1477-1481. http://dx.doi.org/10.1007/s00192-008-0669-9 